\title{
Percepções de gestores de um hospital universitário sobre a qualidade em saúde
}

Perceptions of the managers of a university hospital about quality in health

Percepciones de los gerentes de un hospital universitario sobre la calidad de la salud

\section{Sâmia Jamylle Santos de Azevedo', Larissa Kelly Silva de Moura ${ }^{\mathrm{II}}$, Camila Matias Nascimento ${ }^{\mathrm{III}}$, Theo Duarte da Costa ${ }^{\mathrm{IV}}$, Pétala Tuani Candido de Oliveira Salvador ${ }^{\mathrm{V}}$}

Resumo: Objetivo: descrever as percepções de gestores de um hospital universitário sobre a qualidade em saúde. Método: trata-se de estudo do tipo descritivo, com abordagem qualitativa, realizado em hospital universitário do Nordeste do Brasil. A população de estudo foi composta por 60 gestores. Os dados foram coletados entre $2017 \mathrm{e}$ 2018, a partir de entrevista semiestruturada. Realizou-se análise textual lexicográfica com suporte do software IRaMuTeQ. Resultados: foram analisados 593 segmentos de texto com elucidação das classes: "Influência do modelo de gestão", "Ferramentas de gestão da qualidade", "Concepções de qualidade em saúde" e "Ações de promoção em saúde". Os gestores compreendiam que o modelo de gestão adotado influencia na assistência e, consequentemente, na qualidade do atendimento. Conclusão: verificou-se que os gestores relacionaram a qualidade à resolutividade do atendimento aos usuários, bem como a um bom relacionamento entre profissionais e à consequente melhoria no processo de trabalho.

Descritores: Qualidade da Assistência à Saúde; Gestão da Qualidade; Gestão em Saúde; Hospitais; Gestor de Saúde

Abstract: Objective: to describe the perceptions of managers of a university hospital about quality in health. Method: this is a descriptive study, with a qualitative approach, carried out in a university hospital in Northeast Brazil. The study population was composed of 60 managers. Data were collected between 2017 and 2018, based on a

\footnotetext{
${ }^{\text {I }}$ Enfermeira. Tecnóloga em Gestão Hospitalar. Especialista em Auditoria em Sistemas de Saúde. Universidade Federal do Rio Grande do Norte. Natal, RN, Brasil. E-mail: samiajamylle@hotmail.com ORCID: https:/orcid.org/0000-0002-7178-7379

II Tecnóloga em Gestão Hospitalar. Especialista em Auditoria em Saúde. Universidade Federal do Rio Grande do Norte. Natal, RN, Brasil. Email: larissaksm95@gmail.com ORCID: https://orcid.org/0000-0001-6527-9952

III Tecnóloga em Gestão Hospitalar. Especialista em Auditoria em Saúde. Universidade Federal do Rio Grande do Norte. Natal, RN, Brasil. Email: camila_kemili@hotmail.com ORCID: https://orcid.org/0000-0003-0516-9895

IV Enfermeiro. Doutor em Enfermagem. Universidade Federal do Rio Grande do Norte. Natal, RN, Brasil. E-mail: theodcj@gmail.com ORCID: https://orcid.org/0000-0002-9355-8382

v Enfermeira. Doutora em Enfermagem. Universidade Federal do Rio Grande do Norte. Natal, RN, Brasil. E-mail: petalatuani@hotmail.com ORCID: https://orcid.org/0000-0002-3208-6270
} 
semi-structured interview. Lexicographic textual analysis was performed with the support of the IRaMuTeQ software. Results: 593 text segments were analyzed and classified in the following categories: "Influence of the management model", "Quality management tools", "Conceptions of quality in health" and "Health promotion actions". The managers understood that the management model adopted influences care, and, consequently, its quality. Conclusion: it was found that managers related quality to the problem-solving service provided to users, as well as to a good relationship between professionals and the consequent improvement in the work process.

Descritores: Qualidade da Assistência à Saúde; Gestão da Qualidade; Gestão em Saúde; Hospitais; Gestor de Saúde Descriptors: Quality of Health Care; Quality Management; Health Management; Hospitals; Health Manager

Resumen: Objetivo: describir las percepciones de los gerentes de un hospital universitario sobre la calidad de la salud. Método: estudio descriptivo, con enfoque cualitativo, realizado en un hospital universitario en el noreste de Brasil. La población del estudio consistió en 60 gerentes. Los datos se recopilaron entre 2017 y 2018, a partir de entrevistas semiestructuradas. El análisis textual lexicográfico se realizó con el apoyo del software IRaMuTeQ. Resultados: se analizaron 593 segmentos de texto con las clases: "Influencia del modelo de gestión", "Herramientas de gestión de calidad", "Concepciones de calidad en salud" y "Acciones de promoción de la salud". Los gerentes entendieron que el modelo de gestión adoptado influye en la atención y, en consecuencia, en la calidad de la atención. Conclusión: se encontró que los gerentes relacionan la calidad con la capacidad de resolución de problemas de atención a los usuarios, así como con una buena relación entre los profesionales y la consiguiente mejora en el proceso de trabajo.

Descriptores: Calidad de la Atención de Salud; Gestión de la Calidad; Gestión en Salud; Hospitales; Gestor de Salud

\section{Introdução}

A qualidade é uma temática emergente que vem sendo amplamente discutida no âmbito das organizações de saúde. No contexto de uma exigência pela maior produtividade, trabalhadores e organizações precisam se dedicar ao máximo na busca de um serviço de qualidade, sendo um resultado almejado por todos. A partir disso, percebe-se a mobilização dos serviços de saúde, trazendo o conceito de Gestão da Qualidade como uma forma de gestão de uma instituição, através da participação dos colaboradores, com o objetivo de satisfazer o cliente e beneficiar a todos da organização. ${ }^{1}$

A política da qualidade deve ser um compromisso firmado por escrito, no qual a instituição de saúde se compromete a realizar o atendimento a partir das necessidades dos clientes, almejando a melhoria contínua nos processos e produtos. Para tanto, os colaboradores 
devem ser capacitados e treinados a exercer suas funções de maneira que contemple os objetivos da política. ${ }^{2}$

Nos serviços de saúde, observa-se a importância dessa política para a gestão, visto que atua como metodologia gerencial, como guia para um planejamento em saúde que contemple a avaliação e a busca pela qualidade, o que pode refletir na assistência ofertada ao cliente. ${ }^{3}$ Enfatiza-se, por conseguinte, que para alcançar qualidade é preciso geri-la. Outrossim, é essencial que o gestor se envolva nesse sentido.

Nessa perspectiva, é importante ressaltar que a qualidade é multifatorial, envolve dimensões técnicas e humanas e precisa ser continuamente avaliada. Destaca-se que a avaliação da qualidade em saúde deve abranger elementos da estrutura, do processo e do resultado. A estrutura corresponde às características da assistência em saúde, com relação aos objetivos, recursos físicos, humanos, materiais e financeiros. ${ }^{4}$

O processo compreende as atividades realizadas entre os profissionais e os clientes. Os resultados correspondem ao produto da assistência ofertada, considerando a satisfação dos indivíduos atendidos. ${ }^{4}$ Deste modo, para alcançar a qualidade, é de suma importância o estabelecimento da gestão da qualidade, e o gestor é uma figura essencial nesse processo.

Desse modo, vê-se que as iniciativas para a promoção da qualidade na assistência à saúde em âmbito mundial crescem a cada dia, envolvendo desde a alta direção das organizações até seus funcionários. Portanto, verifica-se a importância de conhecer as percepções e vivências dos gestores nessa temática, isso porque se compreende que pensar na qualidade como elemento basilar da gestão em saúde constitui concepção fundamental para a melhoria do cuidado em saúde. ${ }^{2}$

Além disso, pesquisadores elucidam o papel essencial do gestor de saúde na consolidação de políticas de qualidade nos serviços de saúde, ${ }^{1-3}$ sendo fundamental a investigação do alinhamento da concepção desses sujeitos no que se refere à temática. Em meio a esse processo 
de discussão, estabeleceram-se como questões de pesquisa: Qual a concepção dos gestores de um hospital universitário em relação à qualidade em saúde? Como atuam esses profissionais nos seus espaços de trabalho? Assim, objetivou-se descrever as percepções de gestores de um hospital universitário sobre a qualidade em saúde.

\section{Método}

Trata-se de estudo do tipo descritivo, com abordagem qualitativa. A pesquisa foi realizada no âmbito de um hospital universitário localizado em Natal, Rio Grande do Norte, Brasil. A estrutura de governança deste hospital é formada por um colegiado executivo composto pela Superintendência e três gerências: Gerência de Atenção à Saúde, Gerência de Ensino e Pesquisa e Gerência Administrativa.

A população do estudo foi composta pelos gestores do hospital, tanto da alta cúpula quanto da média gerência, totalizando 73 pessoas. Foi estabelecido como critério de inclusão: ser gestor da unidade de saúde há pelo menos seis meses, tempo considerado adequado para inserção no cotidiano laboral do serviço. Excluíram-se do estudo os profissionais afastados do serviço por quaisquer motivos e aqueles com os quais não se conseguiu agendamento de entrevista após três tentativas, pessoalmente ou via telefone, a depender do setor.

Os 73 gestores foram convidados de forma verbal, pessoalmente ou via telefone, a participar do estudo. Todavia, 13 gestores não foram entrevistados devido à incompatibilidade de agenda - após três tentativas, não se conseguiu agendar a entrevista. Deste modo, a população do estudo totalizou 60 participantes.

Os dados foram coletados entre os meses de novembro de 2017 e abril de 2018, a partir de entrevista semiestruturada, em local e horário previamente combinados com o gestor. As entrevistas foram realizadas por coletador previamente treinado. Utilizaram-se dois instrumentos de pesquisa: 1) formulário de caracterização do participante da pesquisa, com a 
5 | Azevedo SJS, Moura LKS, Nascimento CM, Costa TD, Salvador PTCO

coleta de dados sociodemográficos e profissionais; e 2) guia para realização da entrevista, composto de duas questões abertas - a) O que é qualidade em saúde para você? b) Você utiliza estratégias e ferramentas da gestão da qualidade em seu trabalho? Se sim, quais? O áudio das entrevistas foi gravado, conforme anuência dos participantes da pesquisa. As entrevistas tiveram duração média de 20 minutos.

Os dados resultantes das entrevistas foram transcritos e submetidos à análise textual lexicográfica, com auxílio do software Interface de $R$ por Analyses Multidimensionnelles de Textes et de Questionneires (IRAMUTEQ). Para esta finalidade, utilizou-se a Classificação Hierárquica Descendente (CHD), o que possibilitou a identificação da frequência de cada palavra e sua conexão com as outras, além de auxiliar na análise do corpus textual.

A CHD é um método de análise em que os textos (entrevistas de cada gestor) são analisados de forma lexicográfica. As classes são obtidas a partir do agrupamento de segmentos de texto que, ao mesmo tempo, apresentam vocabulário semelhante entre si, e vocabulário diferente dos segmentos das outras classes. ${ }^{5}$ Assim, as classes representam categorias de análise com vocabulário léxico coerente entre si, representando, portanto, unidades de significação da percepção dos participantes da pesquisa.

Ressalta-se que o estudo seguiu os princípios éticos e legais que regem a pesquisa científica com seres humanos, preconizados nas Resoluções 466/2012, 510/2016 e 580/2018, do Ministério da Saúde, preservando o caráter voluntário dos participantes e o seu anonimato, de modo que se utilizou o termo "gestor" seguido do número da ordem de realização das entrevistas. A pesquisa foi submetida para apreciação ética por Comitê de Ética da instituição proponente e aprovada a partir do Parecer Consubstanciado no 2.151.764, de 3 de julho de 2017, Certificado de Apresentação para Apreciação Ética nº 67253817.9.3001.5292.

\section{Resultados}


Percepções de gestores de um hospital universitário sobre a qualidade em saúde $\mid 6$

Foram analisadas as percepções de 60 gestores acerca da qualidade em saúde, os quais integravam a equipe de um hospital universitário. Destes, $28(46,7 \%)$ eram do sexo masculino e 32 (53,3\%), do sexo feminino, com idades entre 27 e 66 anos. Com relação à formação, observouse uma predominância na área médica $(13 ; 21,7 \%)$, seguida da graduação em Ciências Contábeis (9; 15\%) e Enfermagem $(7 ; 11,7 \%)$.

Predominaram os gestores com especialização (31; 51,7\%), seguidos daqueles com mestrado $(18 ; 30 \%)$, e $52(86,7 \%)$ participantes mencionaram ter realizado algum curso de capacitação na área da gestão. Sobre o tempo em que atuavam como gestores, houve uma variação de 6 meses a 34 anos, e no tempo em que atuavam na instituição, de 9 meses a 37 anos. Por meio da CHD dos depoimentos dos gestores, foram analisados 593 segmentos de texto, retendo $82,0 \%$ do total de textos para elucidação das classes (Figura 1).

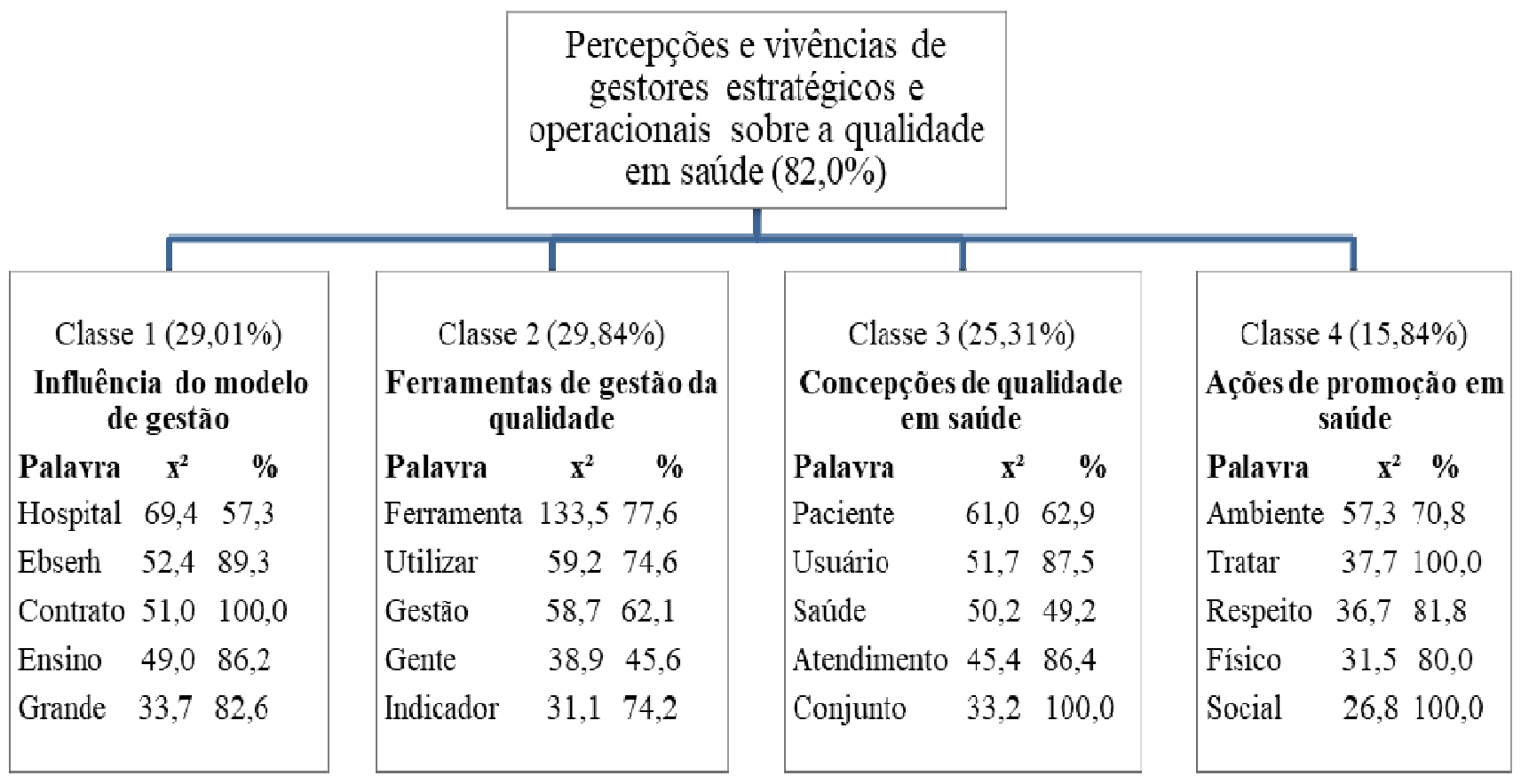

Figura 1 - Dendrograma da Classificação Hierárquica Descendente das percepções e vivências dos gestores sobre a qualidade em saúde. Natal, 2019

*EBSERH - Empresa Brasileira de Serviços Hospitalares 
O vocabulário típico da classe 1 permitiu a contextualização da "Influência do modelo de gestão”, responsável por $29,0 \%$ dos segmentos de texto analisados no âmbito do corpus. As palavras "hospital" e "EBSERH" denotam a concepção dos profissionais sobre a influência do modelo de gestão na promoção da qualidade em saúde, sobretudo, quando há a convivência de funcionários com diferentes vínculos trabalhistas, o que pode influenciar na sensação de “pertencimento à equipe”, que, segundo os gestores, influencia na busca da qualidade em saúde, conforme pode ser observado nos seguintes depoimentos:

Você passa a ter também uma dificuldade de gerir um hospital porque você passa a ter uma convivência de dois grupos de servidores que são de regimes trabalhistas diferentes. (Gestor 1 )

Hoje há uma grande insatisfação de dois grupos diferenciados, um de Regime Jurídico Único e um EBSERH, com exigências e normatizações diferenciadas, geradora de muitos conflitos. (Gestor 30)

Em contrapartida, as palavras "contrato”, “ensino” e "grande” revelam que os gestores se preocupavam com a missão da instituição onde trabalhavam, devendo oferecer ensino, pesquisa e extensão por ser um hospital universitário, de modo que a gestão da qualidade deve englobar também os aspectos relacionados ao ensino, conforme evidenciado por este entrevistado:

Nós temos algumas coisas de qualidade que a universidade pede, temos alguns elementos de qualidade que é a EBSERH que pede e, dentro do contrato de prestação de serviço com o gestor SUS local, nós temos também alguns elementos. (Gestor 1)

É não deixar que o hospital caminhe só para assistência, mas manter a questão do ensino, pesquisa e extensão. (Gestor 4)

À medida que atendemos uma grande variedade de especialidades e com a capacidade que a gente tem como hospital de ensino, para que a gente 
Percepções de gestores de um hospital universitário sobre a qualidade em saúde $\mid 8$

possa realmente cumprir o nosso papel enquanto profissionais da área de saúde, buscamos a qualidade do melhor serviço prestado. (Gestor 62)

A classe 2, “Ferramentas de gestão da qualidade”, representou 29,8\% dos dados textuais analisados e revelou as estratégias utilizadas pelos gestores para consolidar a política de qualidade do hospital. As palavras "ferramenta", "utilizar", "gestão", "gente” e "indicador" denotaram que os gestores utilizavam ferramentas de gestão da qualidade, o que pode ser observado no depoimento a seguir:

Isso tem sido uma busca rotineira de alguns anos, onde a gente tem tentado se apoderar das ferramentas e de uma certa forma disseminar a cultura de segurança e de qualidade. (Gestor 2)

Sobre as ferramentas da gestão da qualidade, a gente faz o planejamento das açôes relacionadas ao setor, a identificação das atividades que proporcionem melhorias e a avaliação dos objetivos alcançados. (Gestor 8)

A estratégia de gestão que nós utilizamos é através da aplicação dos nossos protocolos institucionais, onde utilizamos nossos indicadores para melhorar a qualidade da nossa assistência prestada na instituição. (Gestor 34)

Ao analisar as ferramentas de gestão da qualidade que foram mencionadas como empregadas pelos gestores, destaca-se que $26,6 \%$ dos entrevistados citaram o planejamento como uma das ou a ferramenta de qualidade utilizada e 13,3\% disseram utilizar o Ciclo de Melhoria Contínua. Também foram citadas ferramentas como diagrama de Ishikawa, Matriz SWOT, fluxogramas e diagramas de causa e efeito. A utilização de indicadores e planilhas foi citada como forma de análise interna por $26,6 \%$ dos gestores entrevistados. Também se identificou o uso de softwares que auxiliam a gestão.

Os gestores também mencionaram a capacitação dos profissionais, a busca por um atendimento humanizado e uma boa comunicação dentro da equipe e entre outras equipes e outros setores como ações de gestão que ajudam na busca da qualidade dentro do hospital 
9 | Azevedo SJS, Moura LKS, Nascimento CM, Costa TD, Salvador PTCO

universitário. Contudo, enfatiza-se que 11,6\% dos entrevistados disseram não utilizar nenhuma ferramenta de qualidade no seu trabalho.

A classe 3 foi intitulada "Concepções de qualidade em saúde" e totalizou 25,3\% do corpus analisado. Emergiram como principais palavras dessa classe: "paciente”, "usuário”, "saúde”, “atendimento" e "conjunto". Apreende-se a concepção dos gestores sobre a qualidade em saúde alinhada à ideia da qualidade como elemento multifatorial e decorrente de um processo de trabalho interprofissional, conforme realçado no seguinte depoimento:

Qualidade em saúde representa o resultado de um conjunto de fatores que buscam tornar o processo assistencial seguro, acessivel e pautado no conhecimento científico, com maior índice de evidência e acima de tudo o resultado de um cuidado com bases na humanização ética e responsabilidade. (Gestor 28 )

Qualidade em saúde é um conjunto de ferramentas e estratégias de trabalho voltadas para melhorar o processo de trabalho e dar segurança ao paciente e servidor. (Gestor 31 )

A qualidade em saúde envolve desde um comportamento institucional, de uma qualificação dos profissionais, de uma qualificação dos sistemas de prestação de serviço, seja de sistemas administrativos ou assistenciais, onde o conjunto desse aprimoramento e melhorias seria o produto final de uma assistência adequada ao usuário. (Gestor 64)

Por fim, a classe 4, denominada “Ações de promoção em saúde”, que englobou 15,8\% dos segmentos textuais, destacou os vocábulos "ambiente", "tratar”, "respeito", "físico” e "social”, portanto, elementos que atuam na promoção da saúde do usuário e do trabalhador, de modo que se oferte uma assistência de qualidade. Trata-se de uma concepção de que a satisfação constitui aspecto importante da qualidade em saúde, tanto na perspectiva dos usuários do serviço, quanto de seus funcionários: 
Percepções de gestores de um hospital universitário sobre a qualidade em saúde | 10

A qualidade também envolve o agir em sociedade, ter comportamento ético em relação aos outros, respeito, cordialidade e honestidade, obedecer a princípios éticos que você adquire durante a sua formação. (Gestor 4)

A gente tem que tratar o paciente com todas as condições necessárias em todos os âmbitos do hospital, de forma a proporcionar bem-estar físico e mental. (Gestor 62)

Qualidade em saúde para mim é tudo aquilo que faz com que a gente possa estar proporcionando aos nossos colaboradores para que eles possam estar bem no sentido físico e também no sentido social, porque uma coisa interfere na outra. (Gestor 66).

Outras palavras com destaque na classe 4 foram: “psicológico”, “vida”, “doença” e “relação”. Destacam-se ainda "mental” e “favorável”, que apareceram em 100\% dos segmentos de texto da classe 4 e revelam a preocupação com o trabalhador como aspecto essencial da qualidade em saúde:

Qualidade em saúde é o agente gestor ou colaborador estar sintonizado com a realização de suas demandas profissionais com disposição física e mental de modo a se sentir coautor da efetiva participação junto ao seu cliente ou paciente. (Gestor 30)

Para que a gente consiga desenvolver um trabalho de qualidade é importante que a gente disponibilize também um ambiente favorável. (Gestor 66)

Compreende-se, assim, que as percepções dos gestores sobre a qualidade em saúde perpassa elementos relacionados à gestão, aos funcionários e aos usuários, o que envolve aspectos estruturais (ambiente adequado de trabalho, por exemplo) e do processo de trabalho (como o uso de ferramentas de gestão da qualidade para planejar e avaliar), isso em busca da satisfação de todos e da melhoria da assistência em saúde. 


\section{Discussão}

Em meio à diversidade de profissionais existentes no setor da saúde, o gestor apresentase como peça fundamental para que a realização de ações de qualidade aconteça. Assim, o gestor deve estar primariamente instruído no que concerne ao funcionamento do serviço de saúde em escalas macro e micro, além de ser instrumentalizado para proporcionar um funcionamento adequado dos serviços de saúde, de modo que o conhecimento científico acerca da qualidade em saúde é condição fundamental para a sua gestão. ${ }^{3}$

Todavia, sabe-se que o despreparo técnico científico influencia diretamente o modo de operacionalização das estratégias e a dinâmica das equipes envolvidas nos serviços, o que leva à ineficácia e ineficiência dos processos. ${ }^{6-7}$ Assim, a formação adequada para a gestão da qualidade é essencial ao gestor de saúde, num processo de educação permanente, e pode refletir diretamente na qualidade do serviço.

Compreende-se, assim, que uma instituição em saúde terá outro desempenho a partir do momento em que fizer a escolha de sua gestão por um profissional com conhecimentos técnicos relacionados à administração e domínio de assuntos de saúde. ${ }^{7}$ Nesse sentido, destaca-se que o campo da gestão pautado em processos de avaliação contínuos focados na premissa da qualidade pode auxiliar na tomada de decisões coerentes com a realidade, numa perspectiva de uma gestão eficaz, efetiva e eficiente..$^{1-2}$

Assim, estudos relacionados à gestão da qualidade nos serviços de saúde são emergentes e necessários para uma coesão entre os diversos setores que compõem a área na formulação de práticas eficazes, ao trazer elementos cruciais para uma assistência de qualidade que perpassam a promoção de uma cultura de qualidade. ${ }^{8}$ Atualmente, a cultura de qualidade é definida como o produto de valores, atitudes, competências e padrões de comportamento individuais e de grupo, os quais determinam o compromisso, o estilo e proficiência da administração de uma organização saudável e segura. ${ }^{9}$ 
Percepções de gestores de um hospital universitário sobre a qualidade em saúde | 12

Entretanto, aponta-se que a alta gestão hospitalar ainda é pouco atuante quando as questões envolvem a qualidade em saúde e a segurança do paciente, o que implica em uma cultura desfavorável e no desestímulo dos profissionais de saúde que atuam na linha de cuidado. Por esse motivo, se enfatiza o gestor como fundamental no âmbito da qualidade em saúde, visto que o mesmo é responsável pela promoção de um ambiente aberto de partilha e confiança, para que ele possa conduzir sua equipe por meio da comunicação efetiva, da promoção do desenvolvimento profissional, por meio de capacitações, do incentivo às práticas seguras e da realização do feedback. ${ }^{10}$

As percepções dos participantes da pesquisa mostraram-se, na classe 1, estarem consonantes com a ideia de que o modelo de gestão influencia na assistência e, consequentemente, na qualidade do atendimento. Sobre isso, com o advento da Empresa Brasileira de Serviços Hospitalares (EBSERH), uma empresa pública com o objetivo de administrar unidades hospitalares, foi implantada uma gestão compartilhada, buscando a reestruturação dos hospitais universitários. Todo esse processo foi idealizado no intuito de promover melhorias na gestão, porém culminou com inúmeros conflitos, constituindo intensos debates democráticos com posições a favor e contrárias, principalmente no que diz respeito aos trabalhadores da instituição. ${ }^{11}$

O conflito mais abordado na pesquisa se relaciona ao vínculo trabalhista, pois os trabalhadores das empresas públicas são do regime de trabalho celetista, diferentemente dos demais profissionais, que são regidos pelo Regime Jurídico Único (RJU). Através da Consolidação das Leis Trabalhistas (CLT), o vínculo empregatício é contratual entre empregado e a administração, já os servidores públicos que são regidos pelo regime jurídico único podem ser cedidos à EBSERH, mas o seu regime de trabalho não será alterado. Todos esses fatores atingem diretamente o relacionamento entre esses profissionais e, consequentemente, afetam a 
qualidade no atendimento, ${ }^{12}$ pois o trabalho em equipe é condição fundamental para a qualidade do cuidado em saúde. ${ }^{4}$

O conflito entre os funcionários, nesse panorama, pode se constituir algo preocupante no que concerne a políticas de qualidade em saúde. Isso porque a assistência de média e alta complexidade, mais precisamente na área hospitalar, vem sendo destacada como um local centralizador de problemas na assistência à saúde, que perpassa tanto elementos estruturais quanto de condições de trabalho e, deste modo, recursos humanos alinhados e qualificados quanto às suas ações e concepções tornam-se aspecto fundamental para a qualidade em saúde. ${ }^{13}$

Vale ressaltar que neste ambiente são tomadas decisões capazes de definir o limite entre a vida e a morte das pessoas, tanto no que se refere aos aspectos assistenciais (a decisão por um tratamento, por exemplo) quanto gerenciais (como é o caso da decisão pela compra de um equipamento, por exemplo), acompanhadas de utilização de tecnologias duras e procedimentos de teor mais invasivo, o que gera medo e insegurança para aqueles que fazem uso deste serviço. Desse modo, garantir a qualidade do atendimento desde sua concepção até a sua concretização é primordial. ${ }^{14-15}$

Nesta discussão, pesquisadores discorrem acerca de múltiplas falhas que ocorrem na prestação de cuidados, caracterizadas por insuficiências de processos de trabalhos e estruturas deficitárias, que, uma vez alinhadas, constituem-se caminhos causadores de danos muitas vezes irreversíveis. ${ }^{16}$ Trata-se da discussão sobre a segurança do paciente, uma das dimensões da qualidade em saúde ${ }^{9}$ que vem, cada vez mais, sendo discutida e consolidada no âmbito das instituições de saúde.

Tais consequências advêm de erros ou violações cometidas por pessoas envolvidas na prestação de cuidados (falhas ativas), a partir de decisões relacionadas com o desenho e controle dos processos, bem como suas condições para exercer essas atividades, como insuficiência de recursos humanos, treinamento inadequado, entre outros (falhas latentes). ${ }^{16}$ Essa discussão 
Percepções de gestores de um hospital universitário sobre a qualidade em saúde | 14

perpassa a preocupação dos gestores tanto com os conflitos vivenciados pelos funcionários (classe 1), quanto com a busca da satisfação dos mesmos (classe 4), compreendendo que o funcionário precisa estar implicado na busca da qualidade em saúde.

Portanto, o conceito de qualidade contempla uma tríade necessária para alcançá-la: estrutura, conhecida como recursos disponíveis para efetivação da assistência (físicos, humanos, materiais e financeiros); os processos como as atividades que envolvem profissionais e pacientes, com base em modelos aceitos; e o resultado, que se refere ao produto final da assistência prestada, ao considerar saúde, satisfação de padrões e de expectativas. ${ }^{4,17}$

Destarte, a implantação de sistemas com foco na qualidade requer uma abordagem que reconheça a importância da produção de ações que possibilitem a eficiência, eficácia e efetividade, bem como a otimização dos resultados e a satisfação dos usuários e funcionários. ${ }^{18}$ Essa preocupação com a questão da qualidade constitui parâmetro aludido pelos gestores entrevistados. Revelou-se, com a classe 2, um olhar para os chamados Sistemas de Gestão da Qualidade (SGQs), que vêm crescendo nos últimos anos em várias organizações. O planejamento, a revisão de processos e o acompanhamento de performance, assim como melhorias constantes passaram a ser vitais para o posicionamento da gestão. ${ }^{19}$

A gestão da qualidade é compreendida como um conjunto de ações que abrange os diversos campos da prestação de cuidado (bens e serviços) focadas em desenvolver uma assistência resolutiva, que se apresenta como estratégia fundamental para que os serviços de saúde possam ser ofertados a contento do que rege seus conceitos e definições. ${ }^{3}$ Contudo, essas ações não acontecem de forma aleatória e se faz necessário seguir pontos cruciais para o alcance da excelência na prestação de serviço. Então, é preciso investigar e monitorar os processos por meio de instrumentos pautados nas melhores evidências cabíveis à realidade, analisar o funcionamento das estruturas e definir se os resultados se encontram compatíveis com o esperado. ${ }^{3,18}$ 
15 | Azevedo SJS, Moura LKS, Nascimento CM, Costa TD, Salvador PTCO

Assim, utilizar o planejamento como ferramenta de gestão apresenta-se como impulsionador de práticas voltadas para a articulação dos processos de trabalho, melhorando-se os indicadores de qualidade. ${ }^{20}$ Consonante com esta concepção, na classe 3 verificou-se que a qualidade em saúde e sua avaliação estão pautadas como temas relevantes entre gestores, que objetivam o aperfeiçoamento constante da assistência. No cenário hospitalar, a avaliação é vista como prioritária no âmbito da gestão da qualidade, visto que os fluxos e necessidades do atendimento ao usuário são influenciados diretamente pela mesma. ${ }^{21}$

Em relação ao seu gerenciamento, o gestor dispõe de recursos que o auxiliam no planejamento e gestão da qualidade, como exemplos: Diagrama de Ishikawa, Brainstorming, Indicadores, Diagrama de Causa e Efeito, Ciclo de Melhoria Contínua, entre outros, que, de forma geral, servem como elementos gerenciais de estudo que auxiliam no estabelecimento da melhoria ininterrupta da assistência. ${ }^{3}$ São as chamadas ferramentas de gestão da qualidade, que foram citadas pelos gestores nas classes 2 e 3.

Por fim, na classe 4, evidenciou-se que a promoção da saúde e a qualidade na assistência possuem associação direta, tendo em vista que consistem em método que evidencia com clareza os riscos e danos, no qual seja assegurado o bem-estar dos profissionais e usuários. ${ }^{22}$ Denotouse, assim, que os gestores compreendiam a satisfação como uma importante dimensão da qualidade em saúde.

No âmbito hospitalar, a promoção da saúde, com o objetivo de uma assistência com qualidade tanto para os usuários do sistema como para os trabalhadores, se faz uma atuação indispensável como forma de minimizar ou diminuir os potenciais riscos que possam afetar a saúde dos envolvidos. ${ }^{23}$ Diferentes estratégias podem ser utilizadas para a constante melhoria da oferta da assistência. Através do meio individual, o objetivo está no autoconhecimento e medidas comportamentais; no meio coletivo/organizacional, a execução de palestras, cursos e a educação continuada aos profissionais, bem como a regulação e a aplicabilidade da legislação e 
políticas. Ressalta-se a importância da intersetorialidade na execução das intervenções, de forma que seja abrangente. ${ }^{24} \mathrm{Com}$ isso, a integração entre equipes para oferecer uma assistência qualificada em saúde é fator primordial.

\section{Conclusão}

Desvelou-se que os gestores do hospital universitário estudado relacionaram a qualidade à resolutividade do atendimento aos usuários, bem como a um bom relacionamento entre profissionais e à consequente melhoria no processo de trabalho. Para consolidar a gestão da qualidade em seu ambiente de trabalho, eles têm utilizado ferramentas, com destaque para o uso do planejamento em saúde e de indicadores de qualidade.

Encontraram-se algumas limitações na realização do estudo, como o fato de o estudo retratar uma realidade local. Portanto, os resultados devem ser compreendidos sob esta perspectiva.

Espera-se contribuir com a divulgação e promoção de maior debate da temática estudada, de modo que se possa aprimorar o entendimento dos gestores sobre a qualidade na saúde e as ferramentas da gestão da qualidade, trazendo benefícios na assistência prestada e proporcionando melhorias na gestão em saúde. Além disso, acredita-se que, ao apresentar uma caracterização mais detalhada sobre a atuação dos gestores, bem como suas concepções, o estudo fornece informações que podem subsidiar intervenções importantes para a mudança da realidade, como a discussão permanente da qualidade no âmbito dos serviços de saúde, buscando transformá-la em uma política estratégica que envolva os diferentes níveis de gestão em prol do melhor cuidado em saúde.

\section{Referências}

1. Melo WOS, Maia AES, Barracho AA, Ribeiro BP, Correa CJAG, Correa IG, et al. Gestão da qualidade 
na saúde. UNINGÁ Rev [Internet]. 2014 [acesso em 2020 fev 16];18(1):24-8. Disponível em: http://revista.uninga.br/index.php/uningareviews/article/view/1499

2. Maia ABB, Barbosa AB, Silva MNP, Branco LMGC, Rodrigues LMC, Melo TMTC. Compilação técnico-científica acerca da auditoria e gestão de qualidade: revisão integrativa. Rev Enferm UFPE On Line [Internet]. 2017 [cited from 2020 Feb 11];11:3(Suppl):1489-94. Available from: https://periodicos.ufpe.br/revistas/revistaenfermagem/article/view/13993

3. Silva K, Moreira JAA, Vasconcelos LT, Oliveira LL, Coimbra CK. Gestão da qualidade total nos serviços de saúde: modelo gerencial em desenvolvimento. Rev Eletrônica Gest Saúde [Internet]. 2015 [acesso em 2020 fev 16];6(1):617-32. Disponível em: https://periodicos.unb.br/index.php/rgs/article/view/2589

4. Donabedian A. The definition of quality and approaches to its assessment: explorations in quality assessment and monitoring. Ann Arbor: Health Administration Press; 1994.

5. Camargo BV, Justo AM. Tutorial para uso do software Iramuteq: interface de R pour les analyses multidimensionnelles de textes et de questionnaires [Internet]. 2018 [acesso em 2020 jun 27]. Disponível em: http://iramuteq.org/documentation/fichiers/tutoriel-portugais-22-11-2018

6. Coelho ES, Cortez DCM, Rodrigues CN, Noronha FMF, Santiago LCP. Perfil dos gestores de Unidades Básicas de Saúde em São Luis-MA. Rev Investig Bioméd. 2016;7(1):43-52. doi: 10.24863/rib.v7i1.19

7. Silva LAA, Soder RM, Petry L, Oliveira IC. Educação permanente em saúde na atenção básica: percepção dos gestores municipais de saúde. Rev Gaúcha Enferm. 2017;38(1):e58779. doi: 10.1590/19831447.2017.01.58779

8. Andrade LEL, Lopes JM, Souza Filho MCM, Vieira Júnior RF, Farias LPC, Santos CCM, et al. Cultura de segurança do paciente em três hospitais brasileiros com diferentes tipos de gestão. Ciênc Saúde Colet. 2018;23(1):161-72. doi: 10.1590/1413-81232018231.24392015

9. Sousa P, Mendes W. Segurança do paciente: conhecendo os riscos nas organizações de saúde. Rio de Janeiro: Fiocruz; 2014.

10. Kawamoto AM, Oliveira JLC, Tonini NS, Nicola AL. Liderança e cultura de segurança do paciente: percepções de profissionais em um hospital universitário. Rev Pesq Cuid Fundam [Internet]. 2016 [cited 2010 Jan 20];8(2):4387-98. Available from: http://www.seer.unirio.br/index.php/cuidadofundamental/article/view/4530/pdf_1887

11. Flausino VS. Cultura e poder na organização hospitalar: as relações de poder na implantação da EBSERH em um hospital universitário [dissertação]. Uberlândia: Universidade Federal de Uberlândia; 2015. 135 p. 
Percepções de gestores de um hospital universitário sobre a qualidade em saúde | 18

12. Paula RB. Desafios da gestão do HU/UFSC, após a criação da EBSERH pelo governo federal [dissertação]. Florianópolis: Universidade Federal de Santa Catarina; 2016. 316 p.

13. Mendes JDV, Bittar OJNV. Perspectivas e desafios da gestão pública no SUS. Rev Fac Cienc Med Sorocaba [Internet]. 2014 [acesso em 2020 fev 16];16(1):35-9. Disponível em: https://revistas.pucsp.br/RFCMS/article/view/18597/pdf

14. Silva PL, Paiva L, Faria VB, Ohl RIB, Chavaglia SRR. Triage in an adult emergency service: patient satisfaction. Rev Esc Enferm USP. 2016;50(3):427-32. doi: 10.1590/S0080-623420160000400008

15. Alves KYA, Costa TD, Barros AG, Lima KYN, Santos VEP. Segurança do paciente na terapia intravenosa em unidade de terapia intensiva. Rev Pesq Cuid Fundam. 2016;8(1):3714-24. doi: 10.9789/2175-5361.2016.v8i1.3714-3724

16. Sousa LAO, Fonteles MMF, Monteiro MP, Mengue SS, Bertoldi AD, Dal Pizzol TS, et al. Prevalência e características dos eventos adversos a medicamentos no Brasil. Cad Saúde Pública. 2018;34(4):e00040017. doi: 10.1590/0102-311X00040017

17. Gomes ATL, Alves KYA, Santos VEP. Evidence on the safety of patients as victims of motorbike accidents in the light of Donabedian-proposed model. Rev Cuba Enferm [Internet]. 2017 [cited 2020 Feb 16];33(2):371-85. Available from: https:/www.medigraphic.com/pdfs/revcubenf/cnf-2017/cnf172q.pdf

18. Giovanella L, Mendonça MHM, Fausto MCR, Almeida PF, Bousquat A, Lima JG, et al. Emergency supply of doctors by the Mais Médicos (More Doctors) Program and the quality of the structure of primary health care facilities. Ciênc Saúde Colet. 2016;21(9):2697-708. doi: 10.1590/141381232015219.16052016

19. Lourenço PJF, Cardoso IMMM, Matos FRN, Nodari CH. Cultura organizacional e qualidade em serviços em instituição de saúde pública portuguesa. Rev Pensam Contemp Adm. 2017;11(4):120-35. doi: 10.12712/rpca.v11i4.1001

20. Albuquerque C, Martins M. Indicadores de desempenho no Sistema Único de Saúde: uma avaliação dos avanços e lacunas. Saúde Debate. 2017;41(N Esp):118-37. doi: 10.1590/0103-11042017S10

21. Oliveira JLC, Matsuda LM. Vantagens e dificuldades da acreditação hospitalar: a voz dos gestores da qualidade. Esc Anna Nery. 2016;20(1):63-9. doi: 10.5935/1414-8145.20160009

22. Janini JP, Bessler D, Vargas AB. Educação em saúde e promoção da saúde: impacto na qualidade de vida do idoso. Saúde Debate. 2015;39(105):480-90. doi: 10.1590/0103-110420151050002015

23. Leal LA, Camelo SHH, Rocha FLR, Vegro TC, Santos FC. A promoção da saúde da equipe de enfermagem no âmbito hospitalar. Rev Rene (Online). 2015;16(5):762-72. doi: 10.15253/21756783.2015000500019 
19 | Azevedo SJS, Moura LKS, Nascimento CM, Costa TD, Salvador PTCO

24. Freire RMA, Landeiro MJL, Martins MMFPS, Martins T, Peres HHC. Um olhar sobre a promoção da saúde e a prevenção de complicações: diferenças de contextos. Rev Latinoam Enferm 2016;24:e2749. doi: $10.1590 / 1518-8345.0860 .2749$

Editor Científico Chefe: Cristiane Cardoso de Paula

Editor Associado: José Luís Guedes dos Santos

\section{Autor correspondente}

Pétala Tuani Candido de Oliveira Salvador

E-mail: petalatuani@hotmail.com

Endereço: Escola de Saúde, Universidade Federal do Rio Grande do Norte, Lagoa Nova, Natal, RN, Brasil

CEP: 59078-970

\section{Contribuições de Autoria}

1 - Sâmia Jamylle Santos de Azevedo

Coleta dos dados, análise e interpretação dos dados, redação e revisão crítica.

\section{2 - Larissa Kelly Silva de Moura}

Coleta dos dados, análise e interpretação dos dados, redação e revisão crítica.

\section{3 - Camila Matias Nascimento}

Coleta dos dados, análise e interpretação dos dados, redação e revisão crítica.

\section{4 - Theo Duarte da Costa}

Concepção e planejamento do projeto de pesquisa, coleta dos dados, análise e interpretação dos dados, redação e revisão crítica.

\section{5 - Pétala Tuani Candido de Oliveira Salvador}

Concepção e planejamento do projeto de pesquisa, coleta dos dados, análise e interpretação dos dados, redação e revisão crítica.

\section{Como citar este artigo}

Azevedo SJS, Moura LKS, Nascimento CM, Costa TD, Salvador PTCO. Percepções de gestores de um hospital universitário sobre a qualidade em saúde. Rev. Enferm. UFSM. 2020 [Acesso em: Anos Mês Dia]; vol.10 e95: 1-19. DOI:https://doi.org/10.5902/2179769243697 